\title{
Conductive, Superhydrophobic, and Microwave- Absorbing Cotton Fabric by Dip-Coating of Aqueous Silk Nanofibers Stabilized MWCNTs and Octadecanoyl Chain Bonding
}

Ling Liu Anhui University

Mingyuan Wu

Anhui University

Qingyun Wu

Anhui University

Jiuyi Liu

Anhui University

Jianjun Yang

Anhui University

Jianan Zhang ( $\square$ jianan@ahu.edu.cn )

Anhui University https://orcid.org/0000-0003-3195-9882

\section{Research Article}

Keywords: Multi-walled carbon nanotubes, cotton fabric, conductive, superhydrophobic, microwave absorption

Posted Date: August 31st, 2021

DOl: https://doi.org/10.21203/rs.3.rs-760826/v1

License: (c) (i) This work is licensed under a Creative Commons Attribution 4.0 International License. Read Full License

Version of Record: A version of this preprint was published at Cellulose on April 12th, 2022. See the published version at https://doi.org/10.1007/s10570-022-04538-1. 


\section{Abstract}

A facile dip-coating method to endow cotton fabric (CF) with satisfactory conductivity, superhydrophobicity and microwave absorption performance was proposed based on the combination of multi-walled carbon nanotubes (MWCNTs) incorporation and hydrophobic octadecanoyl chain bonding. The entanglement and bundling of MWCNTs induced by the particularly high aspect ratio and high interaction energy renders homogeneous dispersion of MWCNTs a challenging. The durable coating adhesion of MWCNTs on hydrophilic CF remains the other challenge due to the absence of strong interactions with intrinsic hydrophobic MWCNTs. In this work, silk nanofibers (SNFs) were synthesized by degrading silk at high temperature, which was adopted as dispersant to prepare individually dispersed MWCNTs via ultrasonication and homogenization processes. The coating adhesion of MWCNTs to CF (MWCNTs-CF) was enhanced via dipping coating and thermal treatment induced chemical immobilization cycles. Octadecanoyl chain-tethered MWCNTs-CF (C18-MWCNTs-CF) was manufactured by further treatment with stearoyl chloride to achieve superhydrophobicity. The scanning and transmission electron microscopy micrographs demonstrated that the aggregates of MWCNTs were successfully de-bundled into individually dispersed nanotubes by taking advantages of the high $\pi-\pi$ interaction and electrostatic repulsive interactions between MWCNTs and SNFs. SNFs has the superiority of chemical bonding with $\mathrm{CF}$ at high temperature and providing active sites for subsequent hydrophobic treatment. The electrical conductivity, surface properties, thermal stability, mechanical properties, and microwave absorption performance of the CF samples were evaluated systematically. Compared with pristine CF $\left(1.04 \otimes 10^{10} \Omega\right)$, the C18-MWCNTs-CF exhibited excellent conductive property with surface resistance reaching $55 \Omega$ when the loaded MWCNTs on CF were $247.5 \mathrm{mg} / \mathrm{g}$ in the case of 3 dippingdrying cycles and possessed a relatively greater microwave absorption performance of $-36.08 \mathrm{~dB}$ at 9.28 $\mathrm{GHz}$ with merely $2.7 \mathrm{~mm}$ thickness. Compared with pristine CF, C18-MWCNTs-CF exhibited superhydrophobicity with the WCA increasing from $26^{\circ}$ to $150^{\circ}$ even after 20 scratching cycles due to the combination of facile octadecanoyl group tethering and the increased surface roughness. The biodegradable and recyclable C18-MWCNTs-CF exhibited reasonable electrical conductivity, superhydrophobicity and microwave absorption that promises an ideal application prospect in the field of smart textile and wearable electronic devices.

\section{Introduction}

Cotton fabric (CF) has been extensively applied in clothing, medical care and industrial fabrics for their prominent characteristics such as cellulose recoverability, good mechanical properties, flexibility and air permeability (Gao et al. 2021; Luo et al. 2019). However, the practical application of CF was limited in many fields because of its inherent hydrophilicity and the risk of sparks caused by electrostatic discharge at low temperatures and in humid environments (Su et al. 2020). The bearing of a plenty of hydroxyl groups on the CF surface provides numerous possibilities for subsequent modification through adjusting the surface wettability toward superhydrophobic CF-based composites ( Luo et al. 2019; Lam et al. 2019) Recently, CF combined with functional nanomaterials have attracted enormous attention for their 
considerable application in the fields of flexible wearable devices, smart textiles, strain sensors or health monitoring and so on (Gniotek and Krucińska 2004; Lukowicz et al. 2004; Mahltig et al. 2013).

Due to the superior electrical conductivity and highlighted microwave absorption performance, multiwalled carbon nanotubes (MWCNTs) have become a promising candidate for the preparation of multifunctional CF (Sethi et al. 2017). The design of aqueous MWCNTs dispersant is a crucial necessity to fully utilizing the comprehensive performance of MWCNTs-CF composites in rational balances of strong interfacial interactions between MWCNTs/dispersant and water medium. It is widely accepted that construction of multifunctional CF composites can be realized by combined manipulation of functionalization or mechanical dispersion of MWCNTs (Ma et al. 2010; Ruoff 2018). Currently, the increasing demand for multifunctional CF-based materials in biomedical field is helpful to stimulate study of developing biomolecular-assisted aqueous stripping from bundled MWCNTs to individual MWCNTs. Compared with similar methods using industrialized surfactants and organic solvents, these stripping methods have significant environmental and economic benefits, and also endowed untied MWCNTs with improved biocompatibility and potential functionalization of biomolecules (Anaya-Plaza et al. 2020). A variety of biomolecules, including protein (Liang et al.2020; Fang et al. 2018), peptide (Mann et al. 2020; Geng et al.2019), silk nanofibers (Tan et al. 2020) and nucleator/DNA (Sun et al. 2020) have been applied to realize stripping and aqueous dispersion of defect-free MWCNTs. Silk materials have been utilized to prepare multifunctional composite biomaterials because of their biocompatibility, mechanical properties, adjustable biodegradability, versatile processability (Cheng et al. 2017; Dong et al. 2016; Xiao et al. 2017) and strong interaction between silk proteins and various hydrophobic nanomaterials (such as ferrous oxide, gold and graphene) (Ling et al. 2018; Zhang et al. 2014; Zhao et al. 2020).

It is broadly occurring that construction of superhydrophobic interfacial materials can be realized with the aid of combined manipulation of topography structure and chemical composition of the surface ( Lai et al. 2013). For instance, Xue (Xue et al. 2020) developed a superhydrophobic, flame-retardant and conductive CF via layer-by-layer assembly method based on the combination of poly(ethylenimine), ammonium polyphosphate and MWCNTs. Interestingly, Wang (Shak et al. 2019) provided simple and facile fabrication techniques to load conductive components onto textiles for wearable applications involving dip-coating of single-walled CNTs and polydopamine templating. Zheng (Zheng et al. 2019) designed a conductive superhydrophobic CF by assembling carboxylated and aminated MWCNTs followed by modification with polydimethylsiloxane. Makowski and coworkers (Makowski et al. 2014) presented the findings concerning the preparation and properties of cotton woven fabrics with a conductive network through deposition of MWCNTs on the fiber surface by the padding method. They claimed that the prepared textile could exhibit a high capability under the chosen arrangement of investigations. However, the dispersion of CNTs and the adhesion between CNTs and CF in the above literature required further improvements involving the selecting or designing highly efficient dispersant of MWCNTs and promoting the durability of functional CF composites to meet the practical application. Despite the progress in the multifunctional superhydrophobic textiles, so far, there are few studies about simultaneously endowing CF superhydrophobic, conductive and microwave absorption performance. 
In this work, an efficient strategy for waterborne MWCNTs dispersion stabilized by silk nanofibers (SNFs) was developed. Functional CF composites were fabricated by dip-coating of individually dispersed MWCNTs and surface bonding of octadecanoyl groups. The biocompatible SNFs were prepared from cheap and abundant waste silk and were used as dispersant to prepare individually dispersed MWCNTs. The chemical reaction between active groups of SNF and abundant hydroxyl groups on CF facilitated the enhanced adhesion of MWCNTs towards CF and provide active sites for subsequent superhydrophobic treatment. The conductivity, superhydrophobicity and microwave absorption performance of multifunctional CF composites were investigated. Furthermore, abrasion and tensile tests were carried out to verify the robustness of the modified CF against mechanical forces to evaluate their potential applications in wearable electronic devices, medical care and strain sensors.

\section{Experimental Section}

\section{Materials}

The commercial MWCNTs used in study were purchased from Nanjing XFNANO Materials Tech Co., Ltd (serial number: XFQ041). This kind of MWCNTs has an average diameter of $10-20 \mathrm{~nm}$, length of $20-$ $100 \mu \mathrm{m}$ and purity of $95 \%$. Cotton fabric (cellulose content $=95-98 \%$, length $=25-35 \mathrm{~mm}$, linear density $=2.12-1.56 \mathrm{dtex}$, surface hydroxyl content $=12 \%$ ) and silk was purchased from the local market, Hefei, China. Sodium hydroxide, urea, stearoyl chloride, acetone, ethanol, and triethylamine (TEA) were obtained from Aladdin Industrial Co.; Ltd. Methylene blue was bought from Yashilin Ltd. Co. Unless otherwise noted, all reagents were of analytical grade and used without further purification.

\section{Preparation of silk nanofibers dispersant}

Silk nanofibers were prepared via procedures reported previously (Zhuo et al. 2018). Fresh silk solution obtained by conventional treatment. $20 \mathrm{~g}$ of silk was put into a three-necked flask containing appropriate amount of deionized water under magnetic stirring. Then the stoichiometric sodium hydroxide and urea was added to adjust $\mathrm{pH}$ to $11 \sim 12$. SNFs (5wt\%) was obtained after the silk was hydrolyzed at $90^{\circ} \mathrm{C}$ under alkaline condition.

\section{Aqueous dispersion of SNFs stabilized MWCNTs}

The water dispersion of SNFs stabilized MWCNTs comprised the following specific steps. The MWCNTs and SNFs were added to deionized water with a mass ratio of 1:1. The above mixture was ultra-sonicated at $40 \mathrm{KHz}$ for $30 \mathrm{~min}$ and then homogenized at $12000 \mathrm{rpm}$ for $5 \mathrm{~min}$ at room temperature to obtain SNFs stabilized MWCNTs dispersion with a solid content of $2.6 \mathrm{wt} \%$.

\section{Deposition of MWCNT dispersion on CF}

Pristine CF samples were cut to $3 \times 3 \mathrm{~cm}$ in dimension and washed multiple times with deionized water and ethanol sequentially to remove dust and then dried in an oven at $65^{\circ} \mathrm{C}$ for $6 \mathrm{~h}$. Subsequently, $\mathrm{CF}$ was immerged into MWCNTs dispersion $(5 \mathrm{mg} / \mathrm{mL})$ for $15 \mathrm{~min}$, rinsed with DI water, and then dried at $60^{\circ} \mathrm{C}$. 
The prepared sample was labeled as MWCNTs-CF-n. Four cycles of dipping-drying process were performed to obtain the MWCNTs treated CF, coded as MWCNTs-CF-1, MWCNTs-CF-2, MWCNTs-CF-3, and MWCNTs-CF-4, respectively.

\section{Loading capacity of MWCNTs on pure CF}

$0.1717 \mathrm{~g}$ of pure CF was soaked in the MWCNTs dispersion $(5 \mathrm{mg} / \mathrm{mL})$ for adsorption. The weights of pure CF and MWCNTs-CF-n were recorded as $m_{1}$ and $m_{2}$, respectively. Loading capacity of MWCNTs (MWCNTs/pure CF $(\mathrm{mg} / \mathrm{g})$ ) can be calculated according to the following formula:

$$
\mathrm{LC}=\frac{m_{2}-m_{1}}{m_{1}}
$$

\section{Immobilization of octadecanoyl groups on MWCNTs-CF by esterification}

$2.5 \mathrm{~g}$ of $\mathrm{CF}$ and $3.4 \mathrm{~mL}$ of TEA were dispersed in a flask containing $30 \mathrm{~mL}$ of acetone in ice bath under mechanical stirring followed by the addition of $5.4 \mathrm{~mL}$ of stearoyl chloride. After the solution was stirred in a sealed flask for $1 \mathrm{~d}$ at room temperature, the octadecanoyl groups tethered MWCNTs-CF (C18MWCNTs-CF) was obtained after washing thoroughly with acetone and drying under vacuum overnight for performance tests later.

\section{Microwave absorption measurement}

For electromagnetic parameter measurements, the absorbers were made by mixing the as-prepared SNFsMWCNTs with paraffin at the mass loading ratio of 3:7 and pressed into a coaxial cylinder (3.0 and 7.0 $\mathrm{mm}$, inner and outer diameters). The relative electromagnetic parameters were measured by a vector network analyzer (Agilent N5224A, USA) in the frequency range $2-18 \mathrm{GHz}$. Typically, the performance of microwave absorber significantly depends on their complex permittivity $\left(\varepsilon_{\mathrm{r}}=\varepsilon^{\prime}-\mathrm{j} \varepsilon^{\prime \prime}, \varepsilon^{\prime}\right.$ and $\varepsilon^{\prime \prime}$ represent the ability to store electric and the loss of electric energy), permeability $\left(\mu_{r}=\mu^{\prime}-j \mu^{\prime \prime}, \mu^{\prime}\right.$ and $\mu^{\prime \prime}$ symbolize magnetic field energy and magnetic fields), and impedance matching. Impedance matching is one of the crucial parameters to determine the microwave absorption performance of materials. When $\left|Z_{\text {in }} / Z_{0}\right|$ is equal to 1 , the incident electromagnetic wave is completely absorbed by materials. The microwave absorption performance can be represented by reflection loss $(\mathrm{RL})$ based on the transmit line theory (Li et al. 2017). The RL was calculated using the equations:

$$
\begin{gathered}
\mathrm{RL}=20 \log \frac{z_{i n}-z_{0}}{z_{\text {in }}+z_{0}} \\
Z_{\text {in }}=Z_{0} \sqrt{\frac{\mu_{r}}{\varepsilon_{r}}} \tan h\left(j 2 \pi \frac{f d}{c} \sqrt{\mu_{r} \varepsilon_{r}}\right.
\end{gathered}
$$


where $Z_{i n}, Z_{0}, f, d$, and $c$ are the input impedance, the free space impedance, the microwave frequency, the layer thickness of the testing absorber, and the microwave velocity in free space, respectively ( $\mathrm{Li}$ et al. 2016).

\section{Characterization}

The morphologies of MWCNTs and CF samples were evaluated by scanning electron microscopy (SEM) at $200 \mathrm{kV}$. MWCNTs samples were prepared by drying a diluted suspension (in water) on silicon wafer and CF samples were adhered directly onto conductive tape. The surface chemical compositions of SNFs were investigated by means of Fourier transform infrared spectroscopy (FT-IR) and CF samples were characterized by attenuated total reflectance (ATR) FT-IR. The X-ray photoelectron spectroscopy (XPS) measurement was conducted on ESCALAB 250Xi to analyze valence of elements. The samples were examined by X-ray diffraction (XRD) using a Bruker D8 ADVANCE X-ray diffractometer with Cu Ka radiation $(\lambda=1.54 \AA)$ and a scan range of $2 \theta=10 \sim 70^{\circ}$. The sheet resistance of the CF samples was conducted by a four-point probe meter (SZT-2A, China). The water contact angle (WCA) were performed using a contact angle instrument (DSAIOMKZ, Germany) with deionized water droplets of $5 \mu \mathrm{L}$ at ambient temperature, and the obtained WCA of each sample was the average of five measurements at the different positions. The thermal decompositions of pristine CF, MWCNTs-CF-n, and C18-MWCNTs-CF were studied by thermogravimetric analysis (TGA), which was performed with a STA-449F3 apparatus under $\mathrm{N}_{2}$ with a heating rate of $20^{\circ} \mathrm{C} / \mathrm{min}$.

\section{Results And Discussion}

\section{Fabrication of superhydrophobic, conductive and microwave-absorbing CF}

The synthesis of SNFs and the process of stabilizing MWCNTs in water medium to fabricate a multifunctional CF were briefly introduced in Fig. 1. Aqueous SNFs were obtained after the silk was hydrolyzed with the help of urea under alkaline condition. With the incorporation of SNFs, aqueous dispersion of SNFs stabilized MWCNTs was prepared via ultrasonication and homogenization assisted liquid-phase method. The unwound MWCNTs are stabilized by electrostatic repulsive interactions with SNFs dispersant (Paredes and Villar-Rodil, 2016). Notably, SNFs include aromatic amino acid residues, such as tyrosine, tryptophan, and phenylalanine, which have strong $\pi-\pi$ interactions with the surface of MWCNTs (Liang et al. 2020). The $\beta$-sheet structure, as well as the high negative charge density, endowed SNFs with hydrophobicity and good aqueous dispersibility, allowing the SNFs to act as a surfactant to prevent the restacking of MWCNTs in water (Bai et al. 2014) (Fig. 1a). As shown in Fig. 1b, the placed water droplet wetted the cotton fibers and was absorbed fast representing the obvious hydrophilicity of pristine CF. Subsequently, MWCNTs deposited CF was fabricated by dip-coating and thermal treatment induced chemical immobilization cycles, which were labeled as MWCNTs-CF-n. After further treatment with stearoyl chloride, hydrophobic CF was obtained with the tethered octadecanoyl chains (C18MWCNTs-CF). Compared with the water droplet on the surface of pristine CF, the C18-MWCNTs-CF 
demonstrated an obviously varied wettability and the water droplet maintained a nearly spherical shape exhibiting prominent water repellency.

\section{Structure and morphology of MWCNTs and C18-MWCNTs- CF}

The successful preparation of SNFs dispersant was verified by FT-IR characterization (Fig. 2). Compared with the spectrum of pristine silk (Liu et al. 2017), the characteristic peaks of SNFs were associated to the amide III stretching vibration at about $1290 \mathrm{~cm}^{-1}$, and amide I (C凶O or asymmetric carboxylic group vibration) at around 1689 and $1630 \mathrm{~cm}^{-1}$. Besides, the newly appearing absorption peaks at 3448 and $3351 \mathrm{~cm}^{-1}$ corresponding to $\mathrm{N}-\mathrm{H}$, and the peak of the $1160 \mathrm{~cm}^{-1}$ originating from the existence of $\mathrm{C}-\mathrm{N}$.

To confirm the successful tethering of the octadecanoyl chain, characterization of CF samples was carried out by using ATR FT-IR, which were shown in Fig. 3a. Compared with the spectrum of pristine CF, the newly appearing absorption peaks at 2919 and $2850 \mathrm{~cm}^{-1}$ attributing to $\mathrm{CH}_{2}$ groups from the hydrophobic octadecanoyl groups, and the peaks of the $\mathrm{C} \otimes \mathrm{O}$ stretching vibration at $1816 \mathrm{~cm}^{-1}$ and $\mathrm{C}-\mathrm{H}$ deformation vibration at $1707 \mathrm{~cm}^{-1}$ were also assigned to the existence of octadecanoyl groups. These results confirmed that the octadecanoyl groups were chemically bonded on the CF surface by esterification reactions between active hydroxyl groups of cotton fiber and stearoyl chloride. To further verify the above reaction mechanism, the local chemical composition of the surface was detected by XPS (Fig. 3b). Compared with pristine MWCNTs, the XPS spectrum of the modified CF showed new peaks of N element, indicating that SNFs were successfully introduced on the surface of the modified MWCNTs. After dip-coating of aqueous SNFs stabilized MWCNTs and octadecanoyl chain bonding processes, the appearance of $\mathrm{N} 1 \mathrm{~s}$ and $\mathrm{Cl} 2 \mathrm{p}$ signals testified the successful immobilization of SNFs-MWCNTs and octadecanoyl groups on the surface of CF, respectively. The addition of SNFs on the pristine MWCNTs led to deconvolution of the spectrum into three peaks, namely the original two peaks and a new C\N peak at $286.7 \mathrm{eV}$ (Fig. 3c, d). Moreover, different from the $\mathrm{C} 1 \mathrm{~s}$ peak pattern of pristine $\mathrm{CF}$, the new peaks located at 285.8, 287.2, and $288.1 \mathrm{eV}$ appeared in the $\mathrm{C} 1 \mathrm{~s}$ peak spectra of the C18-MWCNTs-CF, corresponding to the binding energies of $\mathrm{C}-\mathrm{N}, \mathrm{O}-\mathrm{C}-\mathrm{O}$, and $\mathrm{O}-\mathrm{C} \bowtie \mathrm{O}$, respectively (Fig. 3e, f). These XPS analyses are consistent with that of ATR-FTIR results, verifying the successful introduction of SNFs-MWCNTs and octadecanoyl groups.

The X-ray diffraction data collected on the pristine CF, pristine MWCNTs, and C18-MWCNTs-CF samples were shown in Fig. 4. Compared with pristine MWCNTs (Fig. 4a), the characteristic diffraction peak of C18-MWCNTs-CF appeared at $2 \theta=26.06^{\circ}$ corresponding to the (002) crystal plane. The untreated CF and treated CF had the same peak located at $35.2^{\circ}$, which attributed to the crystalline structure of $\mathrm{CF}$, suggesting that crystalline structure of treated CF was not destroyed. The noncovalent interaction between SNFs and MWCNTs preserved the inherent characteristic of MWCNTs backbone and is superior to chemical modification, which weakened the intact structure and electronic properties. It can be 
speculated that the loading of MWCNTs and subsequent octadecanoyl group bonding had not changed the major structure of CF during the preparation of C18-MWCNTs-CF.

The surface morphology of pristine MWCNTs and SNFs-stabilized MWCNTs were investigated via SEM analyses. Without the addition of SNFs dispersant, pristine MWCNTs agglomerated into microsized bundles even after ultrasonication and homogenization processes due to the fact that the commercialized MWCNTs are supplied in the form of heavily entangled bundles (Fig. 5a, b). With the incorporation of SNFs dispersant, microsized MWCNTs bundles disappeared and were scattered into welldispersed MWCNTs with the wrapping of SNFs (Fig. 5c). The $\beta$-sheet structure and the high negative charge density endowed SNFs with hydrophobicity and good aqueous dispersibility, which provided electrostatic repulsive interactions and strong $\pi-\pi$ interactions towards MWCNTs (Bai et al. 2014). The disentangled MWCNTs are stabilized and prevented from the restacking (Liang et al. 2020). With the further enlargement of magnification, the aggregates of MWCNTs were hardly examined (Fig. 5d). As shown in the inset of Fig. $5 \mathrm{~d}$, no precipitation was observed in $5 \mathrm{mg} / \mathrm{mL}$ MWCNTs dispersion whether the sample bottle was placed upside down, which was in sharp contrast to the observation of Fig. $5 \mathrm{~b}$. These results suggested that SNFs was a suitable biomolecule to effectively disperse MWCNTs and efficiently stabilize them in water.

It can be seen that pristine CF presented quite a smooth surface without visible impurities (Fig. 6a, b) (Cheng et al. 2018). Compared with that of pristine CF, the deposition of MWCNTs on the CF surface was presented in the form of gray tubes with a size of approximately 20-30 nm (Fig. 6c, d). It was observed that the connected MWCNTs formed a conductive network owing to the extended percolative structure. With the enlargement of magnification, MWCNTs appeared to be adhered tightly on CF because amino groups of SNFs and hydroxyl groups of CFs reacted with each other during drying process to achieve curing effect (Fig. 6d). After octadecanoyl chain tethering, a mass of grooves emerged on the surface of C18-MWCNTs-CF owing to the detachment of tiny cotton fibers during reaction and stirring processes (Fig. 6e, f). To achieve superhydrophobicity, the key is to construct micro/nano roughed hierarchical surface (Zhang et al. 2017). Compared with the pristine CF, the combination of the enhanced surface roughness induced by MWCNTs disposition and the hydrophobic octadecanoyl group bonding dramatically strengthened the hydrophobicity of C18-MWCNTs-CF. The post-treatment did not destroy the conductive network of MWCNTs in the coating and weakened the adhesion of MWCNTs towards CF surface, which is expected to achieve high conductivity and hydrophobicity.

\section{Properties of multifunctional CF \\ Superhydrophobic durability of CF}

The wetting behavior of pristine CF and C18-MWCNTs-CF were explored using WCA measurements. As displayed in Fig. 7a, the WCA of pristine CF was approximately $26^{\circ}$ and upon approaching, the water droplet was adsorbed completely within $2 \mathrm{~s}$ due to the abundant hydrophilic hydroxyl groups of pristine 
CF. The hydrophilicity of CF was enhanced by the disposition of SNFs stabilized MWCNTs, and the water droplets were quickly absorbed so that the contact angle can't be captured (Movie S1). The octadecanoyl chain-tethered C18-MWCNTs-CF was expected to enable the hydrophilic CF with superhydrophobic characteristics after the active sites of CF and SNFs were reacted with stearoyl chloride. Therefore, C18MWCNTs-CF displayed superhydrophobicity with a WCA of about $154^{\circ}$ due to the increased surface roughness induced by hydrophobic octadecanoyl chain (Fig. 7b and Movie S2) (Fan et al. 2019). The pristine CF was wetted as soon as it was immersed in common contaminated liquids (dyed with methylene blue), and the CF was dyed after being taken out (Fig. 7c1, c2). However, C18-MWCNTs-CF possessed obviously antiwetting performance remarkable towards the examined water (dyed with methylene blue), milk, and coffee due to the superhydrophobicity of C18-MWCNTs-CF (Fig. 7d). Fine sand as pollutant was placed on the surfaces of the C18-MWCNTs-CF and pristine CF. As presented in Fig. 8, in contrast to the pristine $\mathrm{CF}$, water drops falling on the C18-MWCNTs-CF could automatically roll and take away fine sand, demonstrating the remarkable self-cleaning property.

The robustness of medical clothing is required to fulfil the practical application of superhydrophobic interfacial materials. Therefore, the mechanical stability of C18-MWCNTs-CF was explored to evaluate its durability by means of utilizing extreme mechanical force. As presented in Fig. 9a, the C18-MWCNTs-CF was subjected to rubbing and scratching process under external force. After 20 cycles, C18-MWCNTs-CF still possessed superhydrophobicity with a WCA approaching $150^{\circ}$ verifying its mechanical durability (Fig. 9b). Additionally, the stability of C18-MWCNTs-CF was confirmed by repeatedly employing certain stress under the action of axial tensile force. The C18-MWCNTs-CF remained the hydrophobicity with the WCA approximately $148^{\circ}$ after being stretched in case of severe axial tensile force (Fig. 9c). The chemically bonded octadecanoyl groups endowed C18-MWCNTs-CF with excessive abrasion resistance (Wang et al. 2019). Further, the amino and carboxyl groups of SNFs can interact with hydroxyl groups on the surface of CF, which facilitated the strong adhesion of MWCNTs towards CF. The robust superhydrophobicity of C18-MWCNTs-CF are capable of strong repellency to water and effectively prevent water penetration, which efficiently avoid performance deterioration. These results reflected that the microstructure and surface composition of C18-MWCNTs-CF fiber were not damaged during mechanical drawing and thus the mechanical wear stability and long-term durability of superhydrophobic fiber was realized consequently.

\section{Electrical conductivity}

The variation in the loading capacity of MWCNTs on pure CF $(\mathrm{mg} / \mathrm{g})$ under different dipping-drying cycles directly affect the conductivity of the C18-MWCNTs-CF. The loading capacity of MWCNTs as a function of dipping-drying cycle was shown in Fig. 10a. The loading capacity of MWCNTs gradually increased after each dipping-drying cycle and reached the maximum content of $259.2 \mathrm{mg} / \mathrm{g}$ after 4 dipping-drying cycles. As could be observed in Fig. 10b, the sheet resistivity of MWCNTs-CF-n and C18-MWCNTs-CF exhibited similar downward trends at incremental dipping-drying cycles. The sheet resistance of MWCNTs-CF-1 decreased dramatically with the LC of $115.3 \mathrm{mg} / \mathrm{g}(580 \Omega)$ in comparison with that of pristine CF $(2 \times$ 
$10^{10} \Omega$ ). With the dipping-drying cycles increasing to 2 and 3 , the surface resistance of MWCNTs-CF-2 was $187 \Omega$ containing MWCNTs of $203.8 \mathrm{mg} / \mathrm{g}$ and MWCNTs-CF-3 was $40 \Omega$ containing MWCNTs of $247.5 \mathrm{mg} / \mathrm{g}$. But the loading of MWCNTs seemed to be saturated at 3 dipping-drying cycles, as further increase cycle of 4 , the sheet resistivity increased slightly with fluctuation around $40 \Omega$. Therefore, the dipping-drying cycles of CF samples in subsequent studies were 1,2,3. The surface resistivity of MWCNTs-CF-n is slight lower than that of C18-MWCNTs-CF, which may be due to the fact that the adsorbed MWCNTs fell off a little after being stirred in the solvent for $1 \mathrm{~d}$ and washed several times during MWCNTs-CF was modified with stearoyl chloride. The mild decrease in WCA of MWCNTs deposited with CF was ascribed to the certain hydrophilicity of the functionalized MWCNTs, but the superhydrophobicity of the CF was not affected. Furthermore, a test of a bulb lighting experiment was carried out, as shown in Fig. 11. Compared with the pure CF (Fig. 11a), MWCNTs-CF exhibited excellent electrical conductivity and was conducive to lighting the LED at $1.5 \mathrm{~V}$ power supply (Fig. 11b). The strengthened conductivity was originated from the three-dimension electrical paths that were formed by direct contact of MWCNTs.

\section{Thermal stability}

The variation of thermal decomposition and pyrolysis behavior of pristine CF, MWCNTs-CF-n, and C18MWCNTs-CF samples with temperature under nitrogen atmosphere by TGA analysis were presented in Fig. 12. The $T_{5 w t}$ and $T_{\max }$ of MWCNTs-CF-n and C18-MWCNTs-CF moved towards lower temperature compared with that of pristine CF due to the decomposition of SNFs, which confirmed that SNFs stabilized MWCNTs were deposited on CF. Particularly, carbon residue of CF was significantly increased from $5.328 \%$ (pristine CF) to $10.119 \%, 13.370 \%, 13.765 \%$ and $17.581 \%$ of MWCNTs-CF-1, MWCNTs-CF-2, MWCNTs-CF-3, and C18-MWCNTs-CF at $600^{\circ} \mathrm{C}$, respectively. The results of carbon residue were consistent with the increasing LC of MWCNTs on CF, which was conductive to the formation of residue and did not affect the thermal stability. After that, weight loss rate became slower as the remainder was mainly the relatively stable MWCNTs carbon skeleton (Su et al. 2020).

\section{Mechanical property}

The impact of chemical treatments on the mechanical property of CF samples were investigated by monitoring the variation of tensile strength and elongation at break (Fig. 13). Compared with pristine CF, the loading of the MWCNTs layer slightly strengthened tensile strength and elongation at break of MWCNTs-CF-n and C18-MWCNTs-CF. This is because the chemical treatment mainly enhanced the combination of MWCNTs and stearoyl chloride on the CF surface, while the core structure of cellulose cotton (which is mainly responsible for showing mechanical strength) has changed modestly ( Bhattacharjee et al. 2020).

\section{Microwave absorption performance}


Because of its light weight, nanocarbon-based absorbing materials can absorb electromagnetic energy maximally in a wide frequency range, which has gradually become the focus of attention in the field of absorbing waves in the new era (Qi et al. 2020; Zhang et al. 2021). The microwave absorption performance in case of various thickness were characterized by calculating RL values (Fig. 14). Compared with pristine MWCNTs, SNFs-MWCNTs has a relatively greater absorption performance, the $\mathrm{RL}_{\min }$ of SNFs-MWCNTs is $-22.33 \mathrm{~dB}$ as thick as $2.5 \mathrm{~mm}$, while its average $\mathrm{RL}$ is only $-6.57 \mathrm{~dB}$ (Fig. 14a). As shown in Fig. 14b, the $R_{\text {min }}$ of SNFs-MWCNTs were larger than $-20 \mathrm{~dB}$ covering the testing thicknesses from 2.5 to $3.0 \mathrm{~mm}$. Generally speaking, RL values under $-10 \mathrm{~dB}$ indicates $>90 \%$ microwave absorption (Mu et al. 2018) and $>99 \%$ microwave were absorbed when RL is below - $20 \mathrm{~dB}$ (Qi et al. 2016). Obviously, as expected, SNFs-MWCNTs showed the optimum enhanced microwave absorption with the $\mathrm{RL}_{\min }$ for $-36.08 \mathrm{~dB}$ at $9.28 \mathrm{GHz}$ with $2.7 \mathrm{~mm}$ thickness, when the filling amount of MWCNTs was merely $0.78 \%$. The applicable bandwidth of presents the absorption frequency range, which is the critical property for an absorber. The corresponding effective bandwidths over - $10 \mathrm{~dB}$ of SNFs-MWCNTs from 2.5 to $3.0 \mathrm{~mm}$ thickness were calculated to be 3.72 (7.48-11.20), 3.90 (7.16-11.06), 4.00 (6.9610.86), 3.88 (6.81-10.69), 3.88 (6.67-10.55), and $4.16 \mathrm{GHz}(6.47-10.63 \mathrm{GHz})$, respectively. Therefore, SNFs-MWCNTs provided multiple microwave reflection access to obtain advanced microwave absorption performance (Liu et al. 2015), which could be adjusted by facile controlling LC of MWCNTs in the targeted CF composites.

\section{Performance evaluation}

In order to highlight the superiority of as-prepared C18-MWCNTs-CF, a comparison of the C18-MWCNTs$\mathrm{CF}$ in current study with the state-of-the-art of modified cotton fabrics reported in the literature. Compared with the following five similar materials, the greatly enhanced conductivity and superhydrophobicity of C18-MWCNTs-CF were contributed to the efficient SNFs-stabilized MWCNTs and tethered octadecanoyl groups. SNFs can not only disperse MWCNTs effectively, but also have the superiority of chemical bonding with CF at high temperature (the amino and carboxyl groups of SNFs can interact with hydroxyl groups on the surface of CF, which facilitated the strong adhesion of MWCNTs towards CF), further providing active sites for subsequent hydrophobic treatment. As shown in Table 1, C18-MWCNTs-CF displayed the competitive performance that promises an ideal application prospect in the field of smart textile and wearable electronic devices. 
Table 1

Performance comparison of the C18-MWCNTs-CF in current study with the state-of-the-art of multifunctional cotton fabrics reported in the literature.

\begin{tabular}{|c|c|c|c|c|}
\hline Materials & $\begin{array}{l}\text { Conductivity } \\
(\Omega)\end{array}$ & $\begin{array}{l}\text { Superhydrophobicity } \\
\left({ }^{\circ}\right)\end{array}$ & $\begin{array}{l}\text { Microwave } \\
\text { absorption } \\
\text { (dB) }\end{array}$ & References \\
\hline C18-MWCNTs-CF & 40.0 & 154 & -36.08 & This work \\
\hline CO + MWCNTs & 710.0 & 155 & - & $\begin{array}{l}\text { (Makowski et al. } \\
\text { 2014) }\end{array}$ \\
\hline MTMS-MWCNT & $4.0 \times 10^{4}$ & 146 & - & $\begin{array}{l}\text { (Nasirizadeh et } \\
\text { al. 2015) }\end{array}$ \\
\hline C-PDA-CNT & 51.8 & - & - & $\begin{array}{l}\text { (Shak et al. } \\
\text { 2019) }\end{array}$ \\
\hline $\begin{array}{l}\text { (bPEI/CNTs) n } \\
\text { /APP/PDMS }\end{array}$ & 113.3 & 151 & - & (Xue et al. 2020) \\
\hline $\begin{array}{l}\text { CCNT/cotton } \\
\text { composites }\end{array}$ & $2.1 \times 10^{3}$ & - & - & (Li L et al. 2017) \\
\hline
\end{tabular}

\section{Conclusions}

To sum up, superhydrophobic, conductive and microwave-absorbing CF was successfully prepared based on a dip-coating method and surface hydrophobic treatment by depositing SNFs-stabilized MWCNTs and tethering octadecanoyl chain onto CF surface. The LC of MWCNTs loaded on CF achieved $247.5 \mathrm{mg} / \mathrm{g}$ with a WCA of $151^{\circ}$ for 3 dipping-drying cycles as the increased surface roughness of the cotton fiber. And the microwave absorption performance can reach more than $-36.08 \mathrm{~dB}$ when the filling amount of MWCNTs was only $0.78 \%$. This approach was superior to most dispersant-assisted dispersion processes reported to date, the well-dispersed MWCNTs-CF obtained in the process exhibited fewer defects, remarkable antistatic performance $(40 \Omega)$, incremental mechanical property and good fastness even after 20 scratching cycles as compared to pristine CF. Considering the cost, abundant silk, the inexpensive equipment, and absence of organic or toxic solvents and chemicals, SNFs as a stabilizer provided a promising route for large-scale production of MWCNTs dispersion. The multifunctional CF could create intelligent articles with far-ranging applications in sports and work clothing, medical care and strain sensors.

\section{Declarations}

\section{Declaration of Competing Interest}

The authors declare no competing financial interest. 


\section{Acknowledgment}

This work was financially supported by National Natural Science Foundation of China (51973001), the Major Program of University Natural Science Research Project of Anhui Province (KJ2019ZD01), the Key Projects of Innovation Program for Returned Scholars of Anhui Province (2019LCX006), and the Open fund for Discipline Construction, Institute of Physical Science and Information Technology, Anhui University.

\section{References}

1. Anaya-Plaza E, Shaukat A, Lehtonen I (2020) Biomolecule-Directed Carbon Nanotube Self-Assembly. Advanced Healthcare Materials 35:1153-1162

2. Bai S, Zhang XL, Lu Q, Sheng WQ, Liu LJ, Dong BJ, Kaplan DL, Zhu HS (2014) Reversible HydrogelSolution System of Silk with High Beta-Sheet Content. Biomacromolecules 15:3044-3051

3. Bhattacharjee S, Macintyre CR, Wen XY, Bahl P, Kumar U, Chughtai AA, Joshi R (2020) Nanoparticles incorporated graphene-based durable cotton fabrics. Carbon 166:148-163

4. Cheng QQ, Zhang BB, He Y, Lu Q, Kaplan DL (2017) Silk Nanofibers as Robust and Versatile Emulsifiers. ACS Appl Mater Interfaces 9:35693-35700

5. Cheng QY, Liu MC, Li YD, Zhu J, Du AK, Zeng JB (2018) Biobased super-hydrophobic coating on cotton fabric fabricated by spray-coating for efficient oil/water separation. Polymer Test 66:41-47

6. Dong XD, Zhao Q, Xiao LY, Lu Q, Kaplan DL (2016) Amorphous Silk Nanofiber Solutions for Fabricating Silk-Based Functional Materials. Biomacromolecules 17:3000-3006

7. Fan ML, Cheng YP, Tu WM, Zhang HN (2019) Fabrication of nitrogen-doped hollow carbon nanospheres with variable nitrogen contents using mixed polymer brushes as precursors. J Mater Sci 54:8121-8132

8. Fang W, Linder MB, Laaksonen P (2018) Modification of carbon nanotubes by amphiphilic glycosylated proteins. Journal of Colloid and Interface Science 512:318-324

9. Gao D, Li XJ, Li YJ, Lyu B, Ren JJ, Ma JZ (2021) Long-acting antibacterial activity on the cotton fabric. Cellulose 28:1221-1240

10. Geng R, Lu D, Lai Y, Wu SF, Xu ZA, Zhang W (2019) Peptide nanotube for carbon dioxide chemisorption with regeneration properties and water compatibility. Chemical Communications 55:3797-3800

11. Gniotek K, Krucińska I (2004) The basic problems of textronics. Fibres Text East Europe 12:13-16

12. Lai YK, Pan F, Xu C, Fuchs H, Chi LF (2013) In situ surface-modification-induced superhydrophobic patterns with reversible wettability and adhesion. Adv Mater 25:682-1686

13. Lam DV, Won SJ, Shim HC, Kim JH, Lee SM (2019) Turning cotton into tough energy textile via metal oxide assisted carbonization. Carbon 153:257-264 
14. Li L, Fan T, Hu R, Liu Y, Lu M (2017) Surface micro-dissolution process for embedding carbon nanotubes on cotton fabric as a conductive textile. Cellulose 24:1121-1128.

15. Li ZX, Li XH, Zong Y, Tan GG, Sun Y, Lan YY, He M, Ren ZY, Zheng XL (2017) Solvothermal synthesis of nitrogen-doped graphene decorated by superparamagnetic $\mathrm{Fe}_{3} \mathrm{O}_{4}$ nanoparticles and their applications as enhanced synergistic microwave absorbers. Carbon 115:493-502

16. Li ZJ, Hou ZL, Song WL, Liu XD, Cao WQ, Shao XH, Cao MS (2016) Unusual continuous dual absorption peaks in $\mathrm{Ca}$-doped $\mathrm{BiFeO}_{3}$ nanostructure for broadened microwave absorption. Nanoscale 8:10415-10424

17. Liang XP, Li HF, Dou JX, Wang Q, He WY, Wang CY, Li DH, Lin JM, Zhang YY (2020) Stable and biocompatible carbon nanotube ink mediated by silk protein for printed electronics. Advanced Materials 32:200-208

18. Ling SG, Wang Q, Zhang D, Zhang YY, Mu X, Kaplan DL, Buehler MJ (2018) Integration of Stiff Graphene and Tough Silk for the Design and Fabrication of Versatile Electronic Materials. Adv Funct Mater 28: 189-198

19. Liu QH, Cao Q, Zhao XB, Bi H, Wang C, Wu DS, Che RC (2015) Insights into size-dominant magnetic microwave absorption properties of CoNi microflowers via off-axis electron holography. ACS Appl Mater Interfaces 7:4233-4240

20. Liu ZL, Li LQ, Zhao ZY, Liu YP, Lu M (2017) Antistatic silk fabric through sericin swelling-fixing treatment with aminated carbon nanotubes. Materials Science and Engineering: B 22:72-77

21. Lukowicz P, Kirstein T, Tröster G (2004) Wearable systems for health care applications. Method of Information in Medicine 43:232-238

22. Luo XG, Weng W, Liang YX, Hu ZX, Zhang Y, Yang JJ, Yang LJ, Yang SY, Zhu MF, Cheng HM (2019) Multifunctional fabrics of carbon nanotube fibers. Journal of Materials Chemistry A 7:8790-8797

23. Mahltig B, Haufe H, Kim CW, Kang YS, Gutmann E, Leisegang T, Meyer DC (2013) Manganese/TiO composites prepared and used for photocatalytic active textiles. Croat Chem Acta 86:143-149

24. Makowski T, Kowalczyk D, Fortuniak W, Jeziorska D, Brzezinski S, Tracz A (2014) Superhydrophobic properties of cotton woven fabrics with conducting 3D networks of multiwall carbon nanotubes. MWMWCNTs. Cellulose 21:4659-4670

25. Mann FA, Herrmann N, Opazo F, Kruss S (2020) Quantum Defects as a Toolbox for the Covalent Functionalization of Carbon Nanotubes with Peptides and Proteins. Angewandte Chemie International Edition 59: 17732-17738

26. Ma PC, Siddiqui NA, Marom G, Kim JK (2010) Dispersion and functionalization of carbon nanotubes for polymer-based nanocomposites: A review. Composites Part A: Applied Science and Manufacturing 41:1345-1367

27. Mu CP, Song JF, Wang BC, Wen FS, Zhang C, Wang C, Liu ZY, Xiang JY (2018) Facile-synthesized carbonaceous photonic crystals magnetic particle nanohybrids with heterostructure as an excellent microwave absorber. Journal of Alloys and Compounds 741:814-820 
28. Nasirizadeh N, Dehghani M, Yazdanshenas ME (2015) Preparation of hydrophobic and conductive cotton fabrics using multi-wall carbon nanotubes by the sol-gel method. Journal of Sol-Gel Science and Technology 73:14-21.

29. Paredes JI, Villar-Rodil S (2016) Biomolecule-Assisted Exfoliation and Dispersion of Graphene and Other Two-Dimensional Materials: A Review of Recent Progress and Applications. Nanoscale 8:15389-15413

30. Qi QB, Wang Y, Ding XD, Wang W, Xu R, Yu D (2020) High-electromagnetic-shielding cotton fabric prepared using multiwall carbon nanotubes/nickel-phosphorus electroless plating. Applied Organometallic Chemistry 34:5429-5434

31. Qi XS, Hu Q, Cai HB, Xie R, Bai ZC, Jiang Y, Qin SJ, Zhong W, Du YW (2016) Heteronanostructured Co@carbon nanotubes-graphene ternary hybrids: synthesis, electromagnetic and excellent microwave absorption properties. Rep 6:379-386

32. Ruoff R S, Weng W (2018) Strong bundles based on carbon nanotubes. Nature Nanotechnology 13:533-534

33. Sethi J, Sarlin E, Meysami SS, Suihkonen R, Kumar AR, Honkanen M, Keinänen P, Grobert N, Vuorinen $J(2017)$ The effect of multi-wall carbon nanotube morphology on electrical and mechanical properties of polyurethane nanocomposites. Composites Part A: Applied Science and Manufacturing 102:305-313

34. Shak M, Pan JJ, Xu AC, Cheng DS, Cai GG, Wang X (2019) Direct dip-coating of carbon nanotubes onto polydopamine-templated cotton fabrics for wearable applications. Cellulose 26:7569-7579

35. Su MT, Chen XT, Zhang LY, Min J (2020) Synthesis of Active Graphene with Para-Ester on Cotton Fabrics for Antistatic Properties. Nanomaterials 10:1147-1156

36. Sun W, Shen J, Zhao Z, Arellano N, Rettner C, Tang JS, Cao TY (2020) Precise pitch-scaling of carbon nanotube arrays within three-dimensional DNA nanotrenches. Science 368:874-877

37. Tan XX, Wang YQ, Du WH, Mu TC (2020) Top-Down Extraction of Silk Protein Nanofibers by Natural Deep Eutectic Solvents and Application in Dispersion of Multiwalled Carbon Nanotubes for Wearable Sensing. Chem Sus Chem 13:321-327

38. Wang X, Yu, PX, Zhang KM, Wu MY, Wu QY, Liu JY, Yang JJ, Zhang JA (2019)

Superhydrophobic/superoleophilic cotton for efficient oil-water separation based on the combined octadecanoyl chain bonding and polymer grafting via surface-initiated ATRP. ACS Applied Polymer Materials 11:2875-2882Xiao LY, Liu SS, Yao DY, Ding ZZ, Fan ZH, Lu Q, Kaplan DL (2017) Fabrication of Silk Scaffolds with Nanomicroscaled Structures and Tunable Stiffness. Biomacromolecules 18:2073-2079

39. Xue CH, Wu Y, Guo XJ, Liu BY, Wang HD, Jia ST (2020) Superhydrophobic, flame-retardant and conductive cotton fabrics via layer-by-layer assembly of carbon nanotubes for flexible sensing electronics. Cellulose 27:3455-3468

40. Zhang HY, Ma XL, Cao CB, Wang MN, Zhu YQ (2014) Multifunctional Iron Oxide/Silk-Fibroin $\left(\mathrm{Fe}_{3} \mathrm{O}_{4^{-}}\right.$ SF) Composite Microspheres for the Delivery of Cancer Therapeutics. RSC Adv 4:41572-41577 
41. Zhang M, Pang J Y, Bao WH, Zhang WB, Gao H, Wang CY, Shi JY, Li J (2017) Antimicrobial cotton textiles with robust superhydrophobicity via plasma for oily water separation. Appl Surf Sci 419: 1623

42. Zhang WB, Wei LF, Ma ZL, Fan QQ, Ma JZ (2021) Advances in waterborne polymer/carbon material composites for electromagnetic interference shielding. Carbon 31:412-426

43. Zhao GX, Zhang X, Li BC, Huang GY, Xu F, Zhang XH (2020) Solvent-Free Fabrication of Carbon Nanotube/Silk Fibroin Electrospun Matrices for Enhancing Cardiomyocyte Functionalities. ACS Biomaterials Science and Engineering 69:76-84

44. Zheng LZ, Su XJ, Lai XJ, Chen WJ, Li HQ, Zeng XR (2019) Conductive superhydrophobic cotton fabrics via layer-by-layer assembly of carbon nanotubes for oil-water separation and human motion detection. Materials Letters 253:230-233

45. Zhuo HM, Zhang XY, Wang LL, Lu Q, Kaplan DL (2018) Sonication exfoliation of defect-free graphene in aqueous silk nanofiber solutions. ACS Sustainable Chemistry \& Engineering 6:12261-12267

\section{Figures}

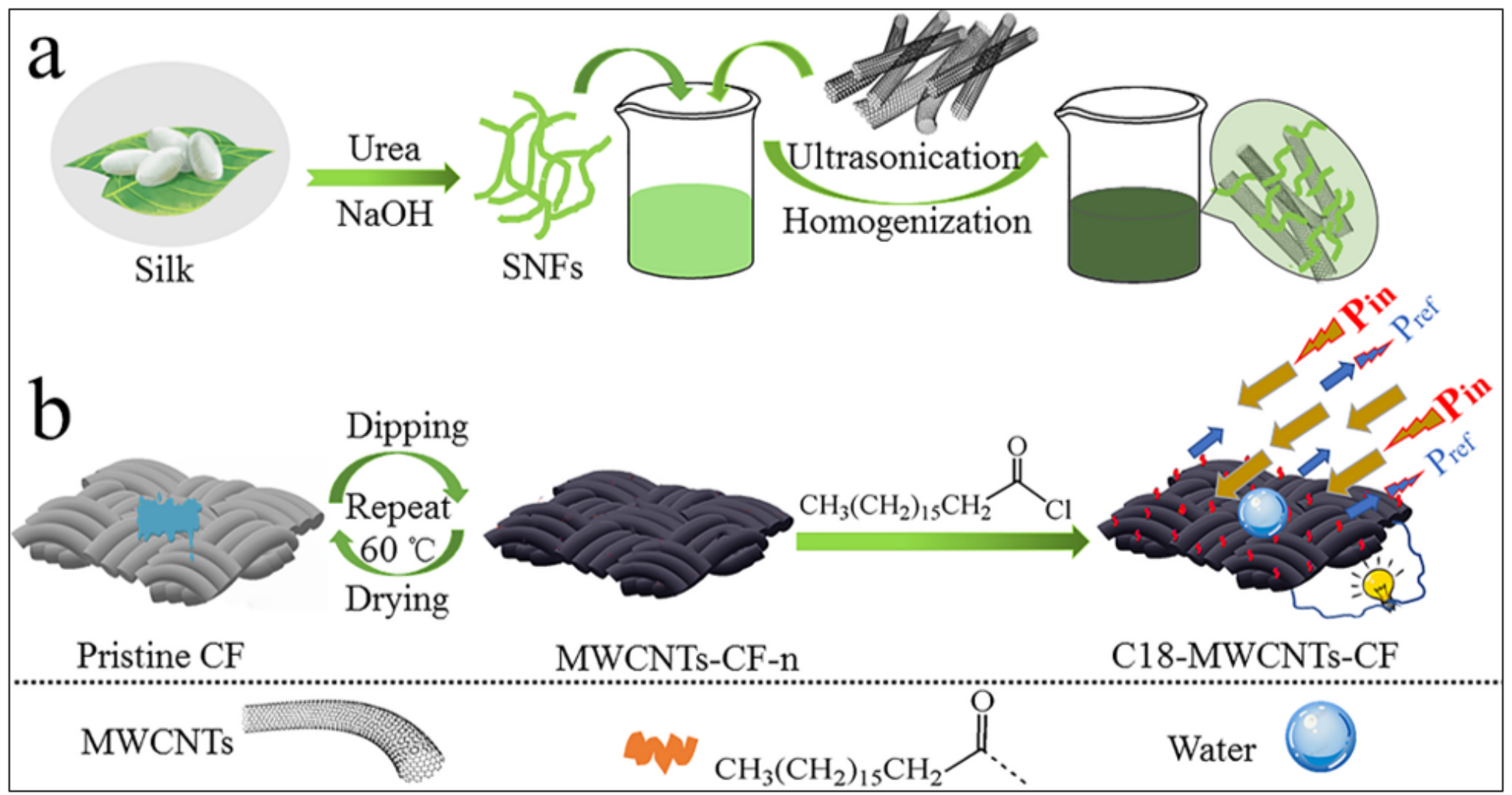

\section{Figure 1}

Schematic illustration of the fabrication of superhydrophobic, conductive, and microwave-absorbing CF. 


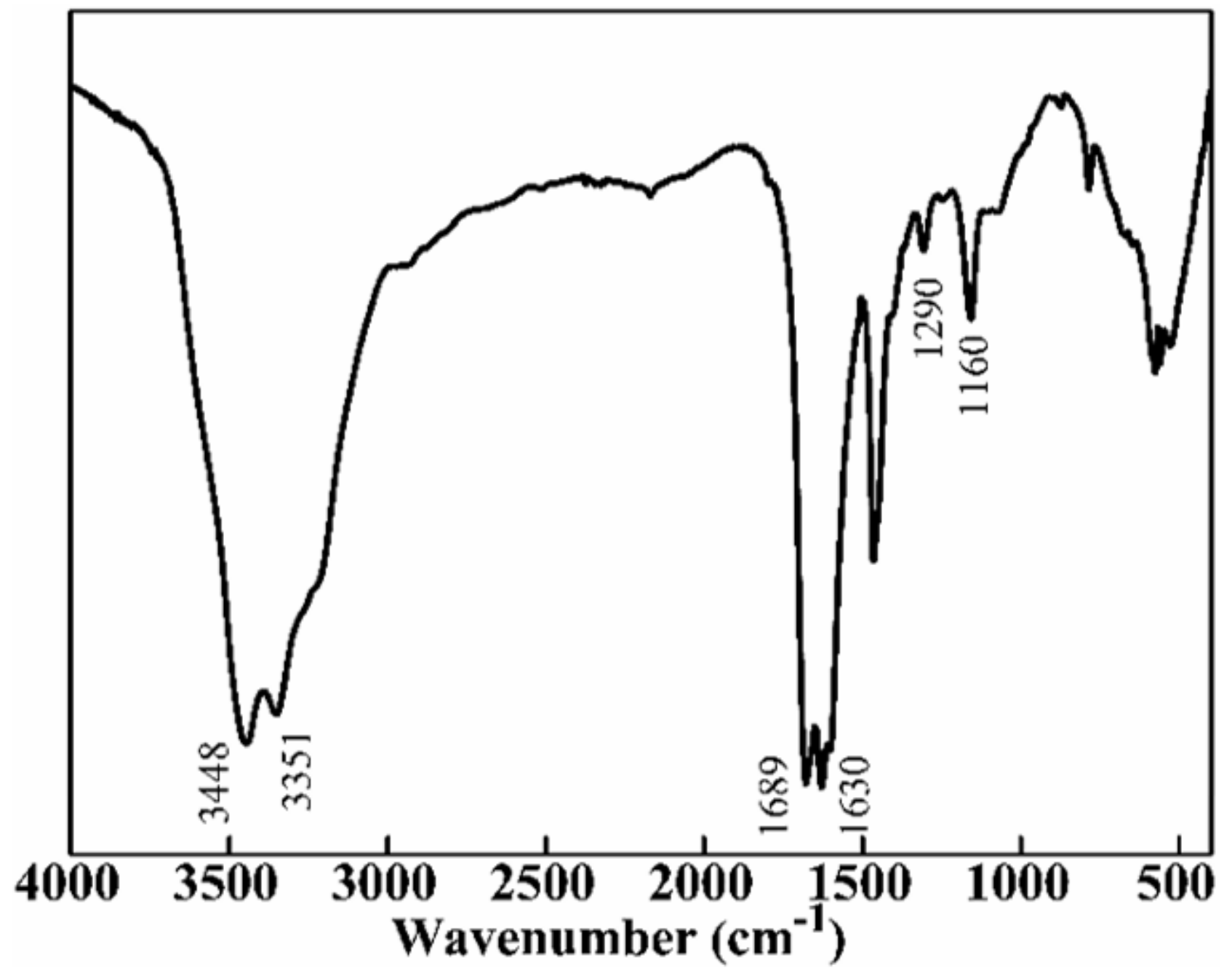

Figure 2

FT-IR spectrum of SNFs. 

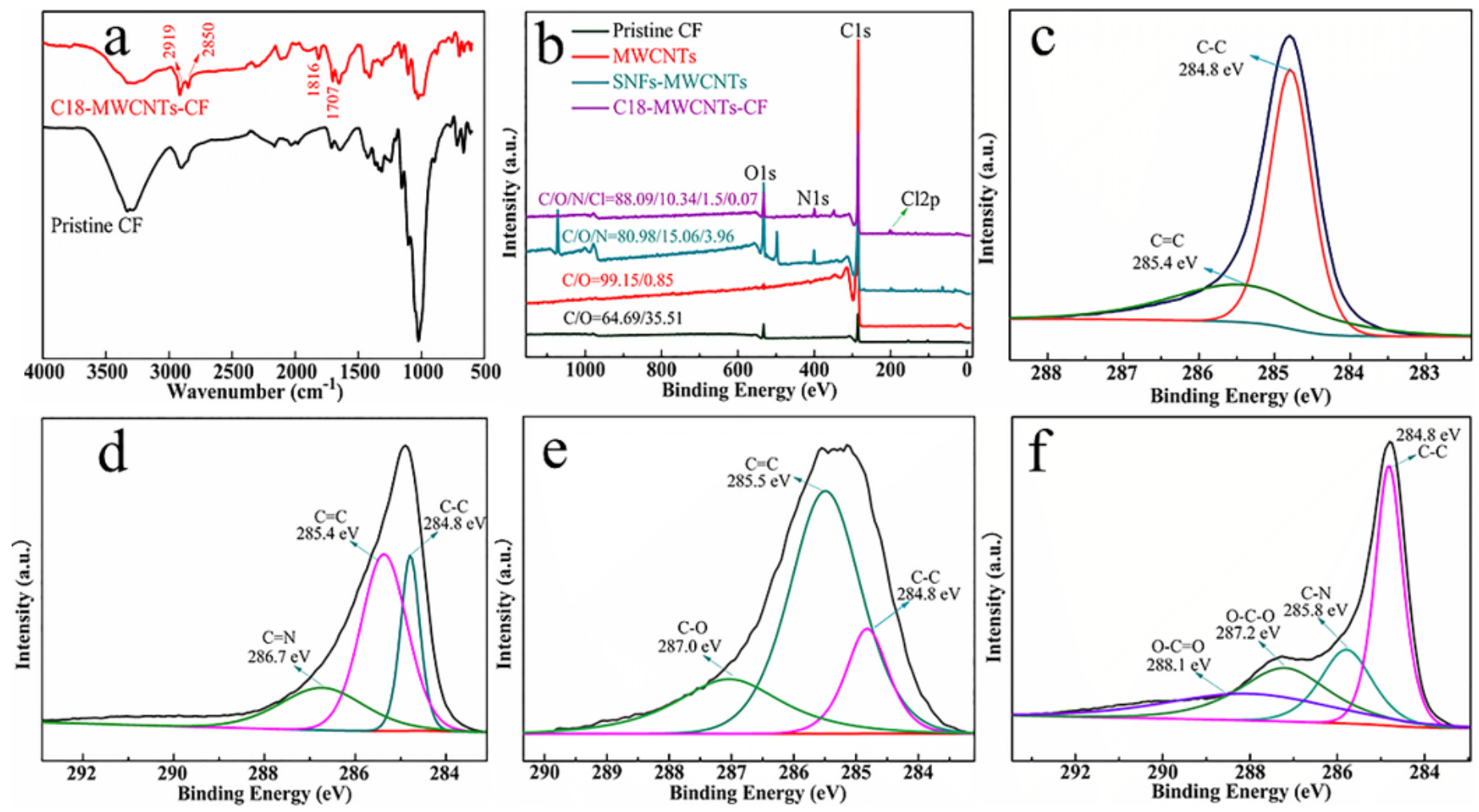

Figure 3

FT-IR spectra of (a) pristine CF and C18-MWCNTs-CF, (b) XPS curves of pristine CF, pristine MWCNTs, SNFs-MWCNTs and C18-MWCNTs-CF, C1s XPS spectra of (c) pristine MWCNTs, (d) SNFs-MWCNTs, (e) pristine $\mathrm{CF}$, and (f) C18-MWCNTs-CF. 


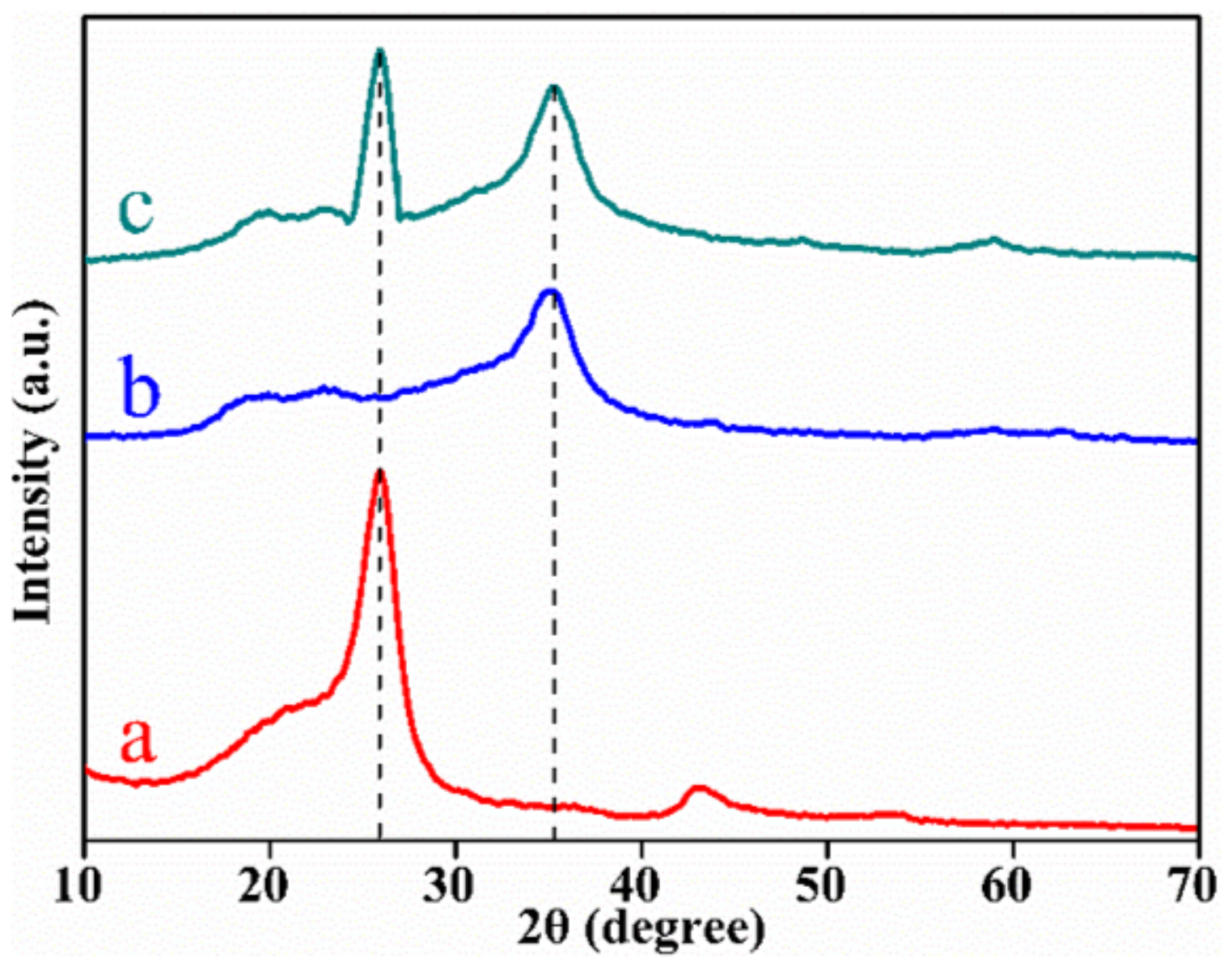

Figure 4

XRD patterns of (a) pristine MWCNTs, (b) pristine CF, and (c) C18-MWCNTs-CF. 

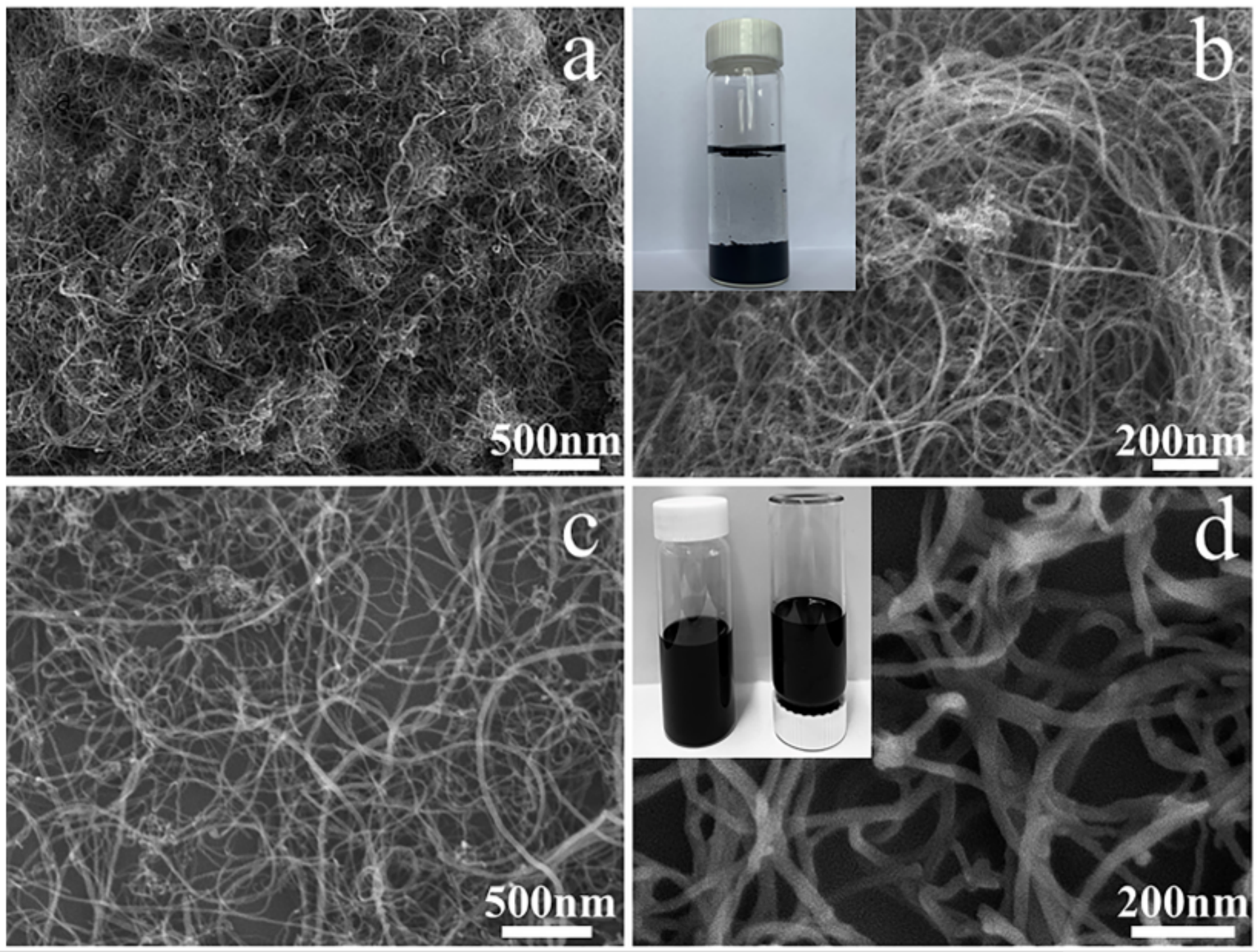

Figure 5

SEM images of pristine MWCNTs $(a, b)$ and SNFs-stabilized MWCNTs with different scale (c, d). And optical photograph of pristine MWCNTs (the inset of $b$ ) and SNFs-stabilized MWCNTs aqueous dispersion (the inset of $d$ ). 

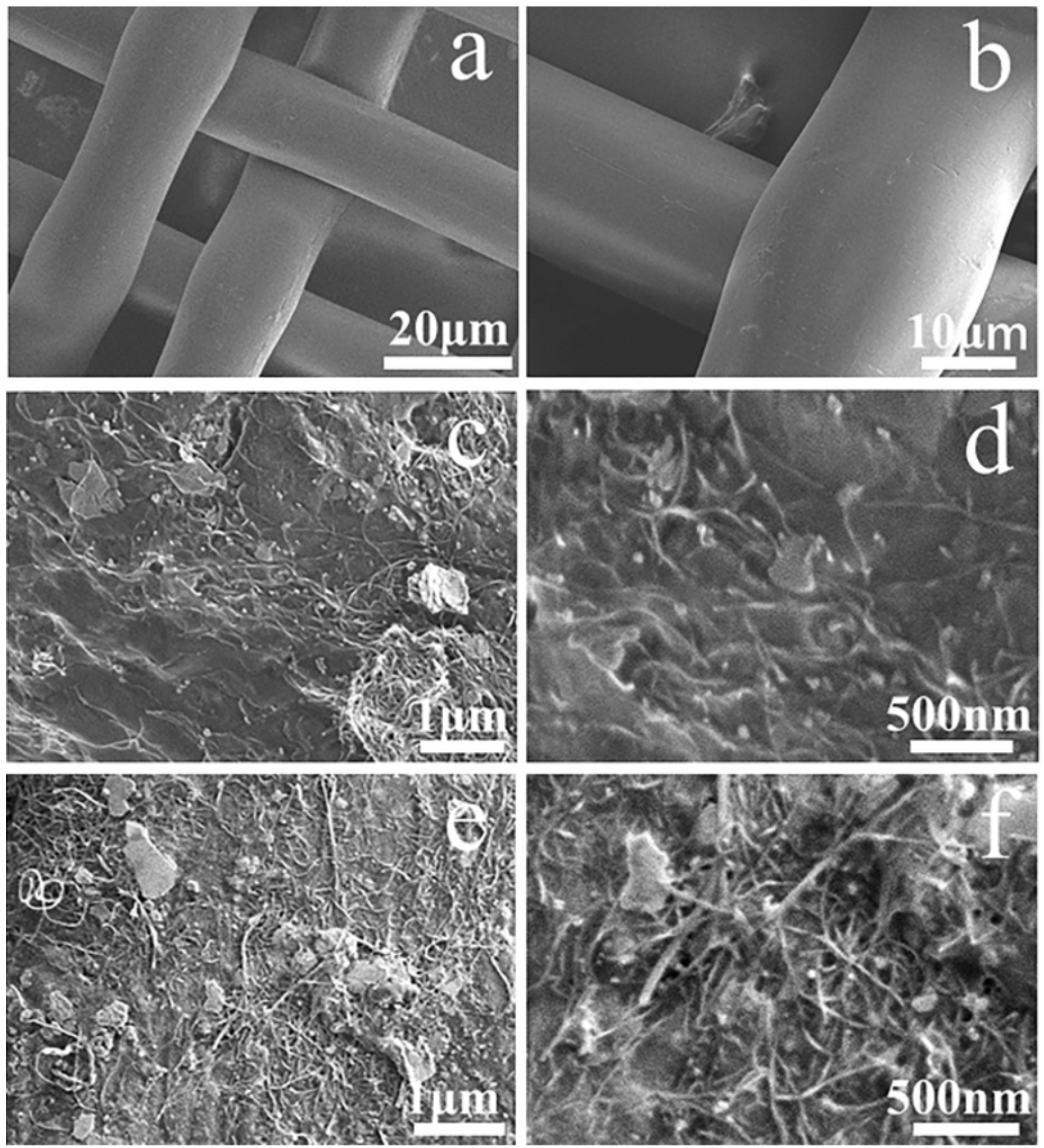

\section{Figure 6}

Representative SEM images demonstrating the morphology changes of $(a, b)$ pristine CF, (c, d) MWCNTs$\mathrm{CF}$, and (e, f) C18-MWCNTs-CF at different magnifications. 


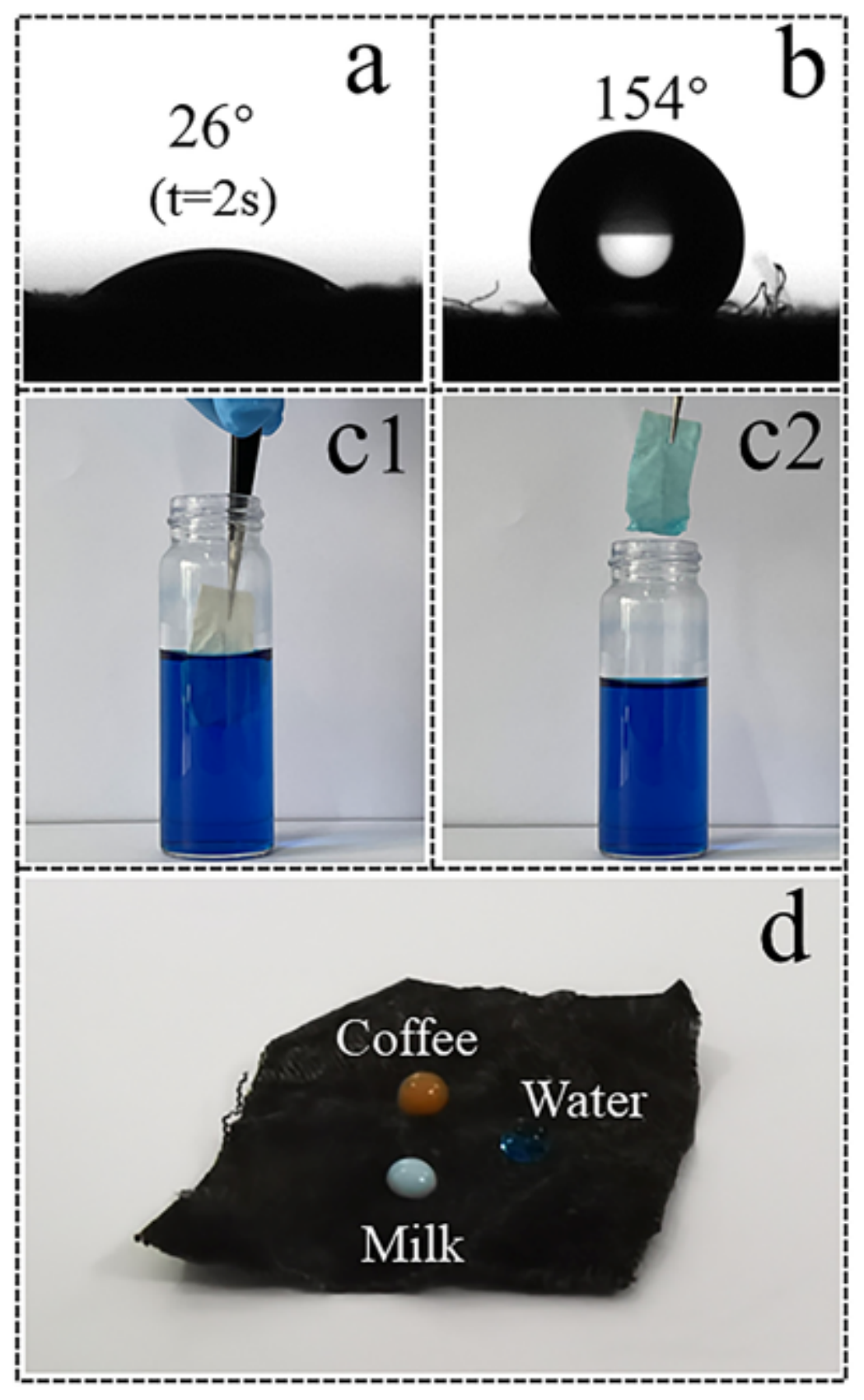

Figure 7

WCA of (a) pristine CF, (b) C18-MWCNTs-CF, (c) and (d) optical picture of water (dyed with methyl blue), milk, and coffee droplets placed on the surface of C18-MWCNTs-CF. 


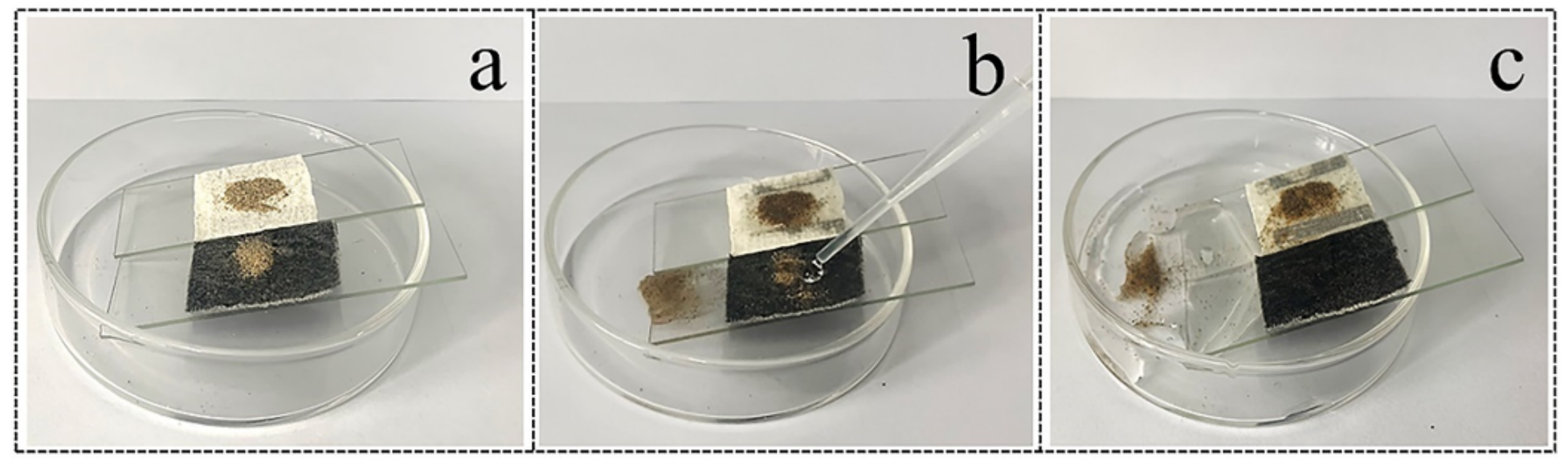

\section{Figure 8}

(a-c) Self-cleaning ability of the C18-MWCNTs-CF.

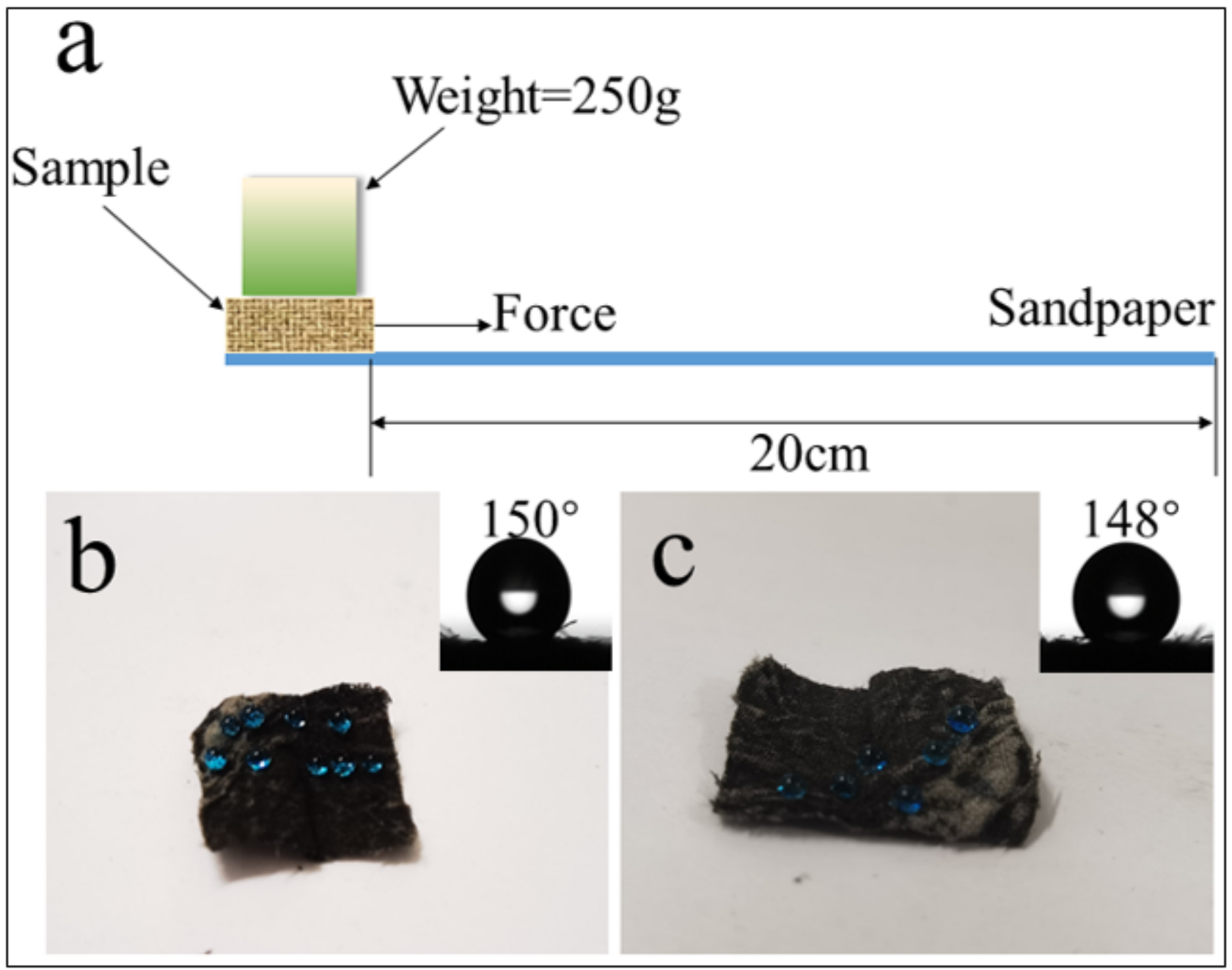

Figure 9

(a) Schematic illustration of the methodology of the abrasion test. Optical photographs of (b) water droplets on C18-MWCNTs-CF after 20 scratching cycles by the sandpaper, and (c) water droplets on the C18-MWCNTs-CF after being stretched by axial tensile forces. Inset shows the corresponding image of 
the WCA of C18-MWCNTs-CF after 20 scratching cycles in part b and the WCA of C18-MWCNTs-CF after being stretched in part $\mathrm{C}$.
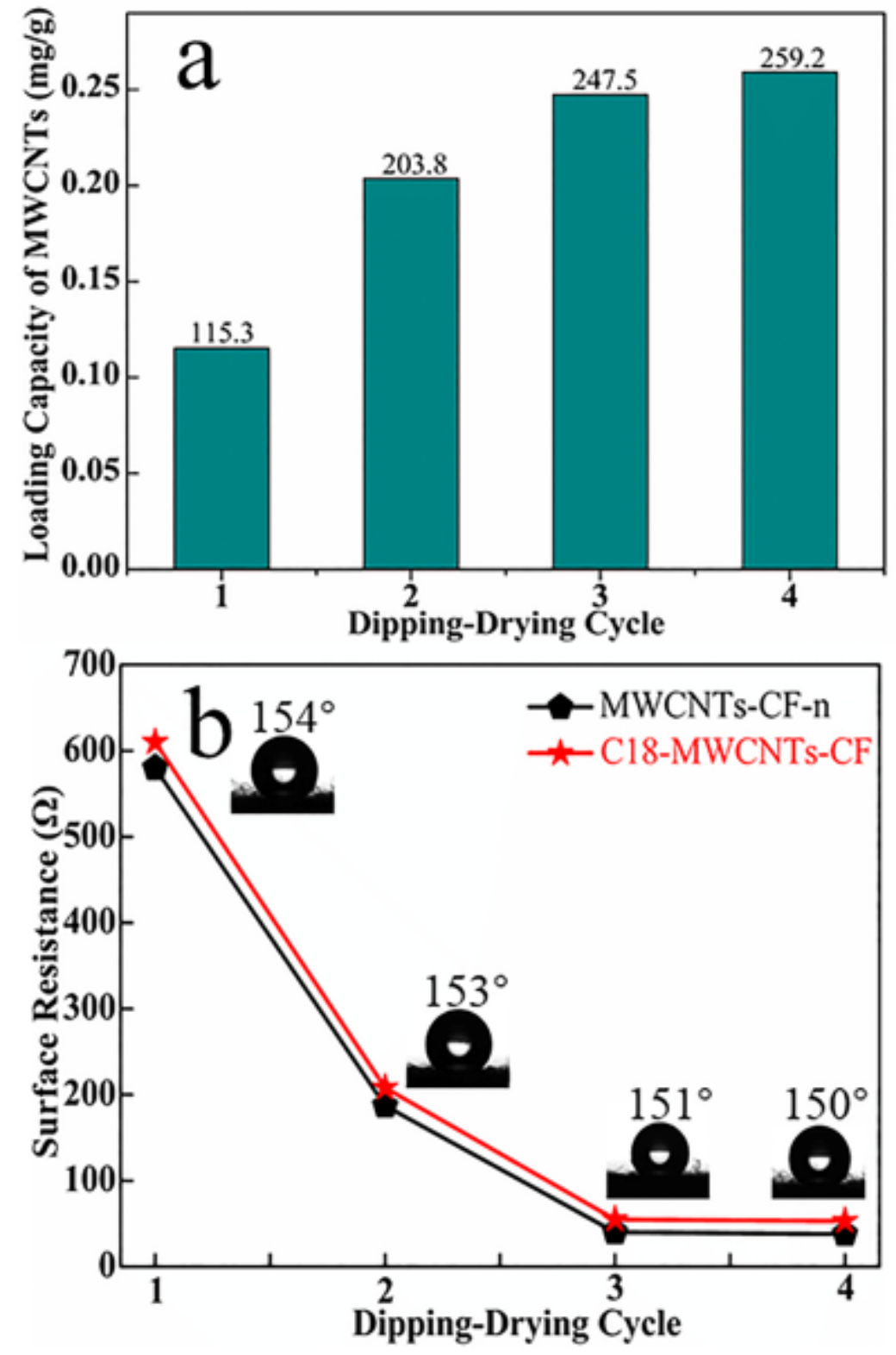

Figure 10

(a) The loading capacity of MWCNTs as a function of dipping-drying cycle and (b) the effect of MWCNTs content on the surface resistivity of CF with a mass ratio of SNFs/ MWCNTs=1:1 (images of WCAs were showed in the inset). 

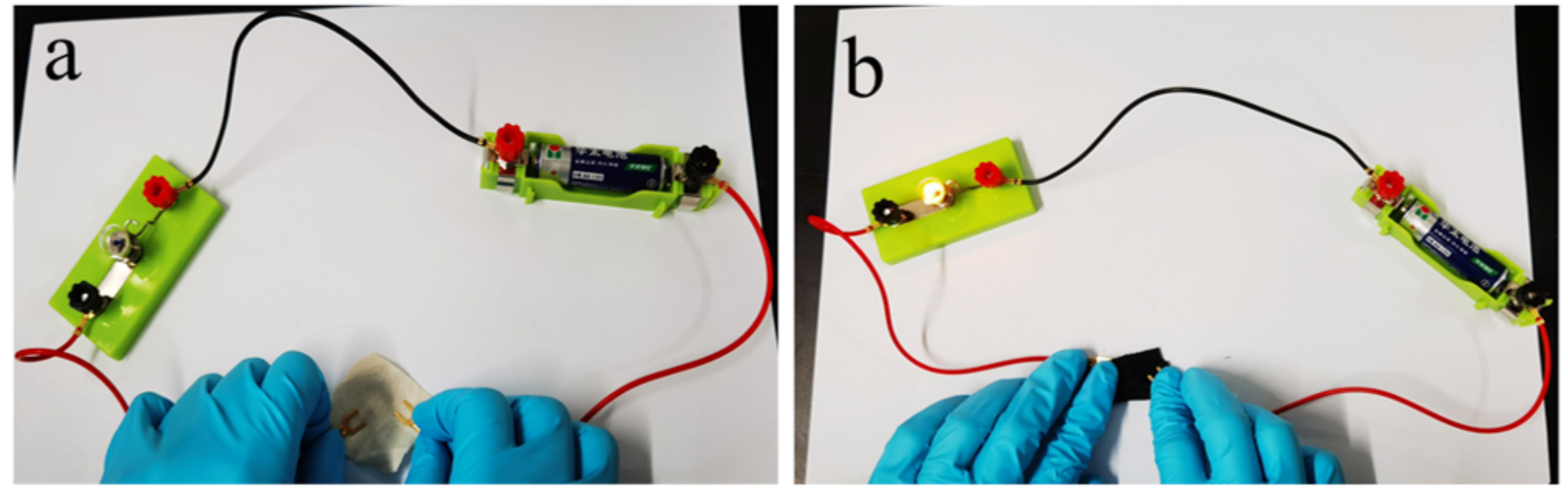

Figure 11

Electrical conductivity test of pure CF (a) and MWCNTs-CF.

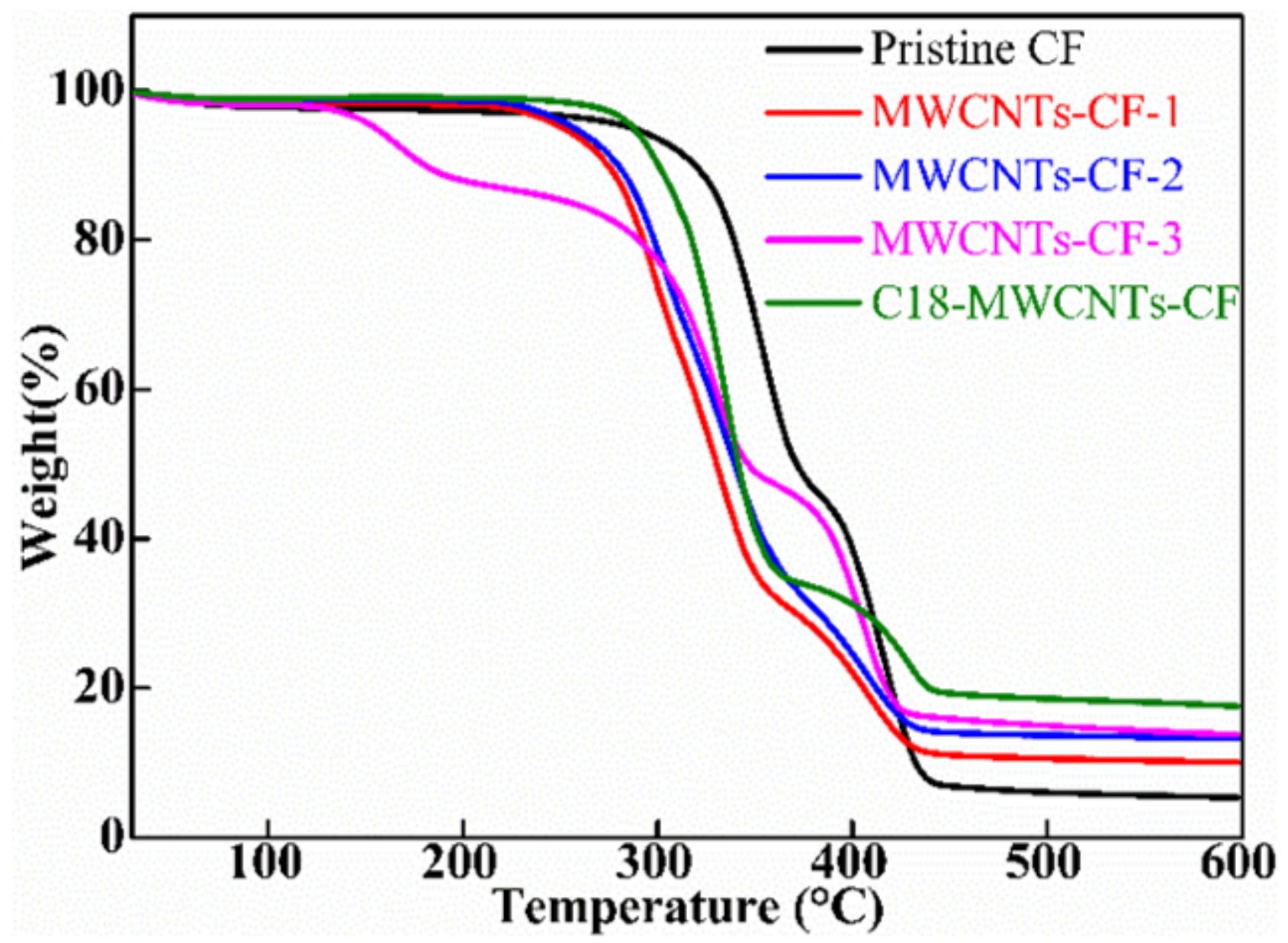

Figure 12

TGA analysis of pristine CF, MWCNTs-CF-n, and C18-MWCNTs-CF in nitrogen atmosphere. 


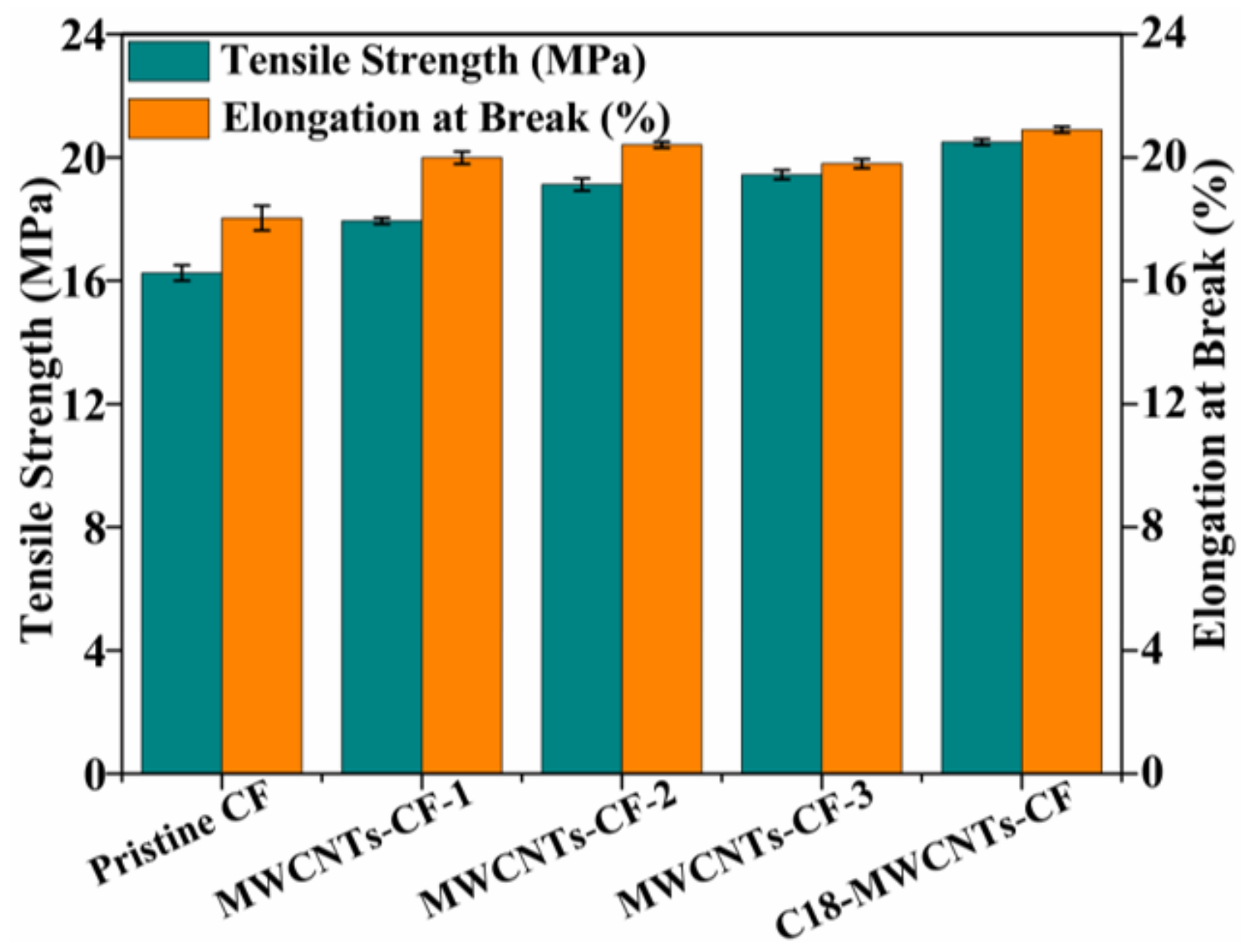

Figure 13

Mechanical properties of pristine CF, MWCNTs-CF-n, and C18-MWCNTs-CF.
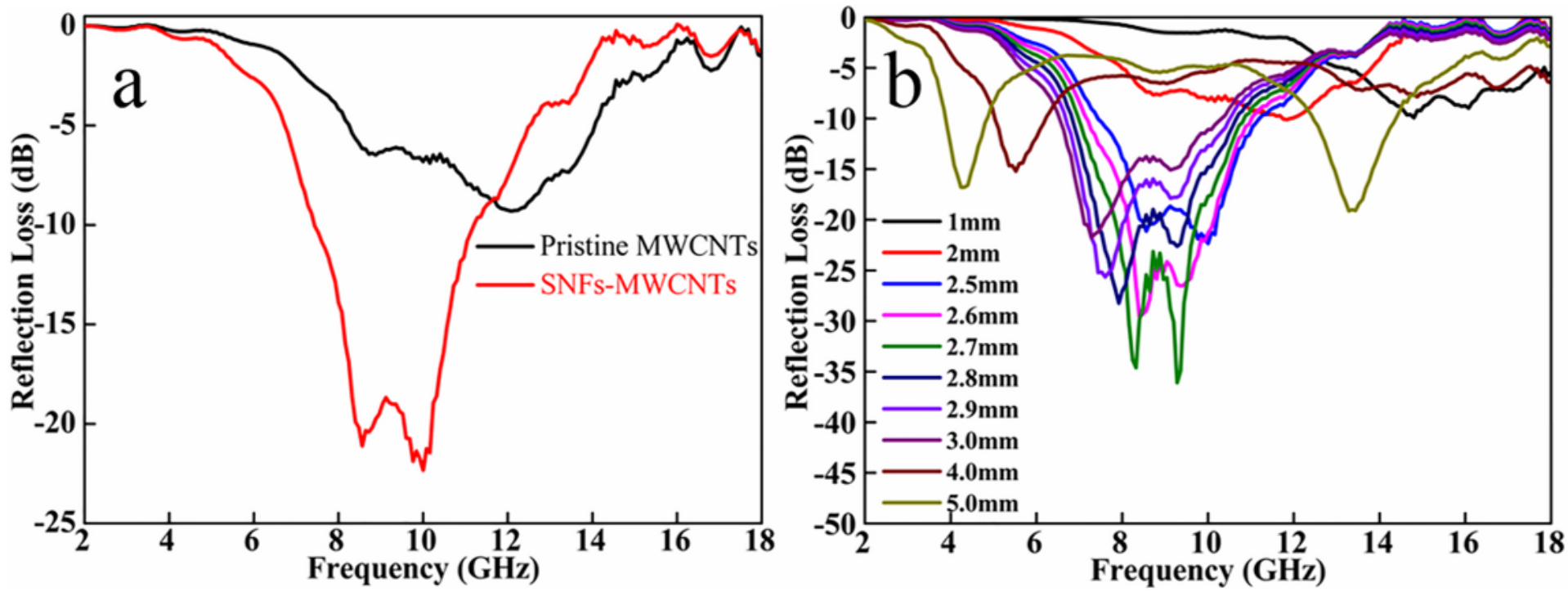

Figure 14 
The microwave RL curves of (a) pristine MWCNTs, SNFs-MWCNTs at $2.5 \mathrm{~mm}$ and (b) SNFs-MWCNTs at different thickness in the frequency range of $2-18 \mathrm{GHz}$.

\section{Supplementary Files}

This is a list of supplementary files associated with this preprint. Click to download.

- GA.tif

- MovieS1.avi

- Movies2.avi

- SupplementaryMateriaIOnline.docx 